\title{
Perfluorooctane sulfonate (PFOS) and other fluorochemicals in viscera and muscle of farmed pigs and chickens in Beijing, China
}

\author{
WANG JieMing, SHI YaLi, PAN YuanYuan \& CAI YaQi* \\ State Key Laboratory of Environmental Chemistry and Ecotoxicology, Research Center for Eco-Environmental Sciences, Chinese Academy of \\ Sciences, Beijing 100085, China
}

Received April 9, 2010; accepted June 18, 2010

\begin{abstract}
Eleven perfluorinated compounds (PFCs) were determined in tissue samples from farmed pigs and chickens $(n=143)$ that were purchased in Beijing in March 2009. The total PFCs mean concentration was the highest in pig liver (3.438 ng g ${ }^{-1}$ wet weight (ww)), followed by pig kidney $\left(0.508 \mathrm{ng} \mathrm{g}^{-1}(\mathrm{ww})\right)$, pig heart $\left(0.167 \mathrm{ng} \mathrm{g}^{-1}(\mathrm{ww})\right)$, chicken liver $\left(0.098 \mathrm{ng} \mathrm{g}^{-1}\right.$ (ww)), chicken heart $\left(0.050 \mathrm{ng} \mathrm{g}^{-1}(\mathrm{ww})\right)$, pork loin $\left(0.018 \mathrm{ng} \mathrm{g}^{-1}(\mathrm{ww})\right)$, and chicken breast $\left(0.012 \mathrm{ng} \mathrm{g}^{-1}(\mathrm{ww})\right)$. Among the PFCs analyzed, perfluorooctane sulfonate (PFOS) was the predominant compound in viscera and muscle of farmed pigs and chickens. In addition, a strong linear correlation $(r=0.932)$ was observed between the concentrations of PFOS and perfluoroundecanoic acid (PFUnDA) in pig liver. Our preliminary conclusion is that there is little potential risk of exposure to PFCs via the consumption of these products in Beijing.
\end{abstract}

perfluorinated compounds, viscera, muscle, dietary intake

Citation: Wang J M, Shi Y L, Pan Y Y, et al. Perfluorooctane sulfonate (PFOS) and other fluorochemicals in viscera and muscle of farmed pigs and chickens in Beijing, China. Chinese Sci Bull, 2010, 55: 3550-3555, doi: 10.1007/s11434-010-4098-y

Perfluorinated compounds (PFCs) are a group of manmade fluorinated organic compounds, which are used extensively in food packaging, textile coatings, lubricants, surfactants, fire-fighting foams, and nonstick coatings for cookware $[1,2]$. Concern about the environmental persistence and potential toxicity of PFCs has grown over the past several decades [2]. Consequently, research has increasingly focused on PFCs and their effects on environmental pollution, toxicology and food safety. These compounds are widely distributed in air, dust, water, soil, wild animals, and humans [3-7]. In 2000, the main manufacturer of perfluorooctane sulfonate (PFOS) in the United States, 3M, voluntarily phased-out its production. However, PFOS is still produced in China and annual production has increased in recent years [2]. In May 2009, the Conference of the Parties 4 (COP-4) of the Stockholm Convention classified PFOS and perfluorooctane sulfonyl fluoride as persistent

*Corresponding author (email: caiyaqi@ rcees.ac.cn) organic pollutants.

PFOS can bind to proteins [8] and is mainly distributed in liver, blood serum, kidney, heart and muscle [9]. PFOS can bioaccumulate and biomagnify through the food chain [10-12]. The environmental distribution of a suite of PFCs, including PFOS and perfluorocarboxylates, has been investigated in the food web of the bottlenose dolphin [11]. Trophic magnification factors for PFOS and some perfluorocarboxylates indicated biomagnification occurred in the marine food web. Another study indicated that accumulation of PFOS was comparable with that of short and medium chained fatty acids [13].

In recent years, the occurrence of PFCs in human serum has attracted much attention [14,15]. Research has also increasingly focused on the routes of human exposure, including food, water, air, and indoor dust [16-22]. Tittlemier et al. [18] showed that humans are mainly exposed to PFOS through food consumption, which accounts for $60 \%$ of exposure. Furthermore, the levels of PFCs in meat products 
were found to be relatively high among all the foodstuffs analyzed. High levels of PFOS have also been found in the liver of both wild and farmed animals in Japan [23]. While PFCs have been detected in various meat products from some countries [18,20,24], very little information is available on the PFCs in these products from China.

Beijing is the political and cultural capital of China and a center of international activity. By 2008, the population of Beijing had exceeded 20 million. Pork and chicken are the main meats consumed by Chinese people and account for up to $88 \%$ of meat consumption among pork, chicken, beef, and mutton [25]. The aim of the present study was to investigate the levels of PFOS and 10 other fluorochemicals in meat products (farmed pigs and chickens) readily available in Beijing. The data could be used for a pilot exposure assessment of consumption of these products.

\section{Materials and methods}

\subsection{Samples}

In March 2009, pig hearts, pig livers, pig kidneys, pork loin, chicken hearts, chicken livers and chicken breast samples were purchased randomly from 14 markets in eight districts in downtown Beijing, China (Figure S1). All the samples were kept at $-20^{\circ} \mathrm{C}$ in their original packages until analysis.

\subsection{Chemicals}

Perfluoroheptanoic acid (PFHpA, >98\%), perfluorooctanoic acid (PFOA, 95\%), perfluorononanoic acid (PFNA, 97\%), perfluorodecanoic acid (PFDA, 97\%), perfluorododecanoic acid (PFDoDA, 96\%), perfluorotetradecanoic acid (PFTA, $96 \%$ ), 2-perfluorooctyl ethanoic acid (FOEA, >98\%), and 2H-perfluoro-2-decenoic acid (FOUEA, >98\%) were purchased from Alfa Aesar (Lancashire, England). Perfluoroundecanoic acid (PFUnDA, 95\%), potassium salts of perfluorohexane sulfonate (PFHxS, $\geqslant 98 \%$ ) and PFOS $(\geqslant 98 \%)$ were obtained from Sigma-Aldrich (Oakville, ON, Canada). Sodium perfluoro-1-[1,2,3,4- $\left.{ }^{13} \mathrm{C}_{4}\right]$ octanesulfonate (MPFOS, $\geqslant 98 \%$ ) and perfluoro-n-[1,2,3,4- $\left.{ }^{13} \mathrm{C}_{4}\right]$ octanoic acid (MPFOA, $\geqslant 98 \%$ ) were purchased from Wellington Laboratories (Canada) for use as internal standards.

Tetra- $n$-butylammonium hydrogen sulfate (TBA) was purchased from Acros Organics (Morris Plains, NJ, USA). Methyl tert-butyl ether (MTBE) was purchased from J. T. Baker (Phillipsburg, NJ, USA). Methanol (HPLC grade) was obtained from Fisher Scientific (Pittsburgh, PA, USA). Ammonium acetate $\left(\mathrm{NH}_{4} \mathrm{OAc},>97 \%\right)$ and ammonium hydroxide (analytical grade; 50\%, v/v) were purchased from Alfa Aesar (Ward Hill, MA, USA). Potassium hydroxide (super grade, $>98 \%$ ) and sodium carbonate (super grade, $\geqslant 99.8 \%$ ) were obtained from the Beijing Chemical Reagent Factory (China).

All water $\left(>18.2 \mathrm{M} \Omega \mathrm{cm}^{-1}\right)$ used in this study was pre- pared with a Milli-Q Advantage A10 system (Millipore, USA).

\subsection{Analytical method}

Two different modified extraction methods, ion-pairing and alkaline digestion [26], were used for viscera samples (pig heart, pig liver, pig kidney, chicken heart, and chicken liver) and muscle samples (pork loin and chicken breast), respectively. Before extraction, samples were thawed and homogenized. For chicken heart, at least 20 samples were pooled and homogenized as one extraction sample, while each chicken liver extraction sample was composed of at least three samples. For viscera samples, a 1-g aliquot was taken and placed in a polypropylene (PP) centrifuge tube, which was precleaned with methanol. Internal standards (MPFOS and MPFOA) were added into the tube, followed by $1 \mathrm{~mL}$ of $0.5 \mathrm{~mol} / \mathrm{L} \mathrm{TBA}$ solution and $2 \mathrm{~mL}$ of buffer $(\mathrm{pH}$ $10,0.25 \mathrm{~mol} / \mathrm{L}$ sodium carbonate). After mixing, $5 \mathrm{~mL}$ of MTBE was added, and the mixture was shaken for $20 \mathrm{~min}$ at $250 \mathrm{r} / \mathrm{min}$. The organic and aqueous layers were separated by centrifugation at $3000 \mathrm{r} / \mathrm{min}$ for $15 \mathrm{~min}$. Then, 4 $\mathrm{mL}$ of MTBE was removed and transferred to a second 15-mL PP tube. The extraction was repeated twice as described above, except that $5 \mathrm{~mL}$ of MTBE was removed each time instead of $4 \mathrm{~mL}$ in the subsequent extractions. All three extracts were combined. The final extract was concentrated to $1 \mathrm{~mL}$ under nitrogen gas after the addition of $1 \mathrm{~mL}$ of methanol. For muscle samples, $1 \mathrm{~g}$ of each sample was freeze-dried and ground into powder before extraction. The sample was spiked with internal standards and extracted by shaking for 16 hat $250 \mathrm{r} / \mathrm{min}$ with $10 \mathrm{~mL}$ of $10 \mathrm{mmol} \mathrm{L}^{-1}$ $\mathrm{KOH}$ in methanol in a PP tube, and then centrifuged. The supernatant was transferred into a PP tube and the residue was extracted with $3 \mathrm{~mL}$ of methanol. The supernatants were combined and concentrated to $1 \mathrm{~mL}$ under nitrogen gas. Each sample (viscera or muscle) was diluted to $40 \mathrm{~mL}$ using water in a PP container and loaded onto a Waters Oasis ${ }^{\circledR}$ WAX single-use cartridge $(6 \mathrm{cc} / 150 \mathrm{mg})$, which was pre-conditioned with $4 \mathrm{~mL}$ of methanol and $4 \mathrm{~mL}$ of water. The sample was loaded onto the pre-conditioned cartridge with a positive pressure pump. The SPE cartridge was washed with $4 \mathrm{~mL}$ of acetate buffer solution ( $\mathrm{pH} 4$ ) and 4 $\mathrm{mL}$ of methanol, and eluted with $2 \% \mathrm{NH}_{4} \mathrm{OH}$ in methanol (target fraction). The flow rate of all steps was controlled at 1 drop s $^{-1}$. The eluate was concentrated to $1 \mathrm{~mL}$ under nitrogen for injection. Blanks of methanol (HPLC grade), treated as real samples in all steps, were processed with each batch.

Analysis of 9 perfluorinated acids and 2 fluorotelomer acids was performed using a high performance liquid chromatography (HPLC, P680 pump, Ultimate 3000 autosampler, Dionex, Sunnyvale, CA, USA) electrospray ionization tandem mass spectrometry (ESI-MS/MS, API 3200, Applied Biosystems/MDS SCIEX, USA). The experimental 
conditions for ESI-MS/MS are shown in Table S1. A $10 \mu \mathrm{L}$ aliquot was injected into a Dionex Acclaim $120 \mathrm{C} 18$ column (5 $\mu \mathrm{m}, 4.6 \mathrm{~mm}$ i.d. $\times 150 \mathrm{~mm}$ length). Methanol (A) and $50 \mathrm{mmol} \mathrm{L}^{-1} \mathrm{NH}_{4} \mathrm{OAc}(\mathrm{B})$ were delivered at a flow rate of

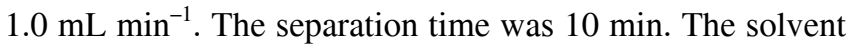
gradient started at $28 \% \mathrm{~B}$, was ramped over 4 min to $5 \% \mathrm{~B}$, held for $3 \mathrm{~min}$, increased to $28 \% \mathrm{~B}$, and held at this level until $10 \mathrm{~min}$.

\subsection{Quality assurance and data analysis}

Quantification was performed using the internal standard method. A 10-point standard calibration curve was constructed using serial dilutions from $0.01-50 \mu \mathrm{g} \mathrm{L}^{-1}$ and fit with a $1 / x^{2}$ weighted regression. MPFOS and MPFOA were used as internal standards. The limits of detection (LODs) were calculated as three standard deviations from the average concentration in the extraction blanks. LODs were in a range of $0.002-0.032 \mu \mathrm{g} \mathrm{L}{ }^{-1}$ (Table S2). The correlation coefficient was $>0.99$ for each analyte. Recoveries of 11 spiked PFCs and two internal standards are shown in Table $\mathrm{S} 2$. The standard calibration curves were conducted before analysis and mixed standard ( $2 \mathrm{ng}$ ) was run as the quality control concentration, which was measured after every 10 injections to check for instrumental drift. If the standards were not within $\pm 20 \%$ of their initial values, a new calibration curve was constructed.

To minimize the background, all potential sources of instrumental and procedural contamination were eliminated. Polytetrafluorethylene (PTFE) materials were removed from the laboratory equipment. All the containers employed during the sample preparation and analysis procedures were washed thoroughly with methanol prior to use. One method blank sample was analyzed with every batch of eight samples. All analytes detected in the method blanks were below the LOD.

For samples where the compounds were not detected, the levels were treated as zero in statistical analysis. Data were analyzed by SPSS 13.0 (SPSS Inc., Chicago, IL, USA, 2005). Spearman's correlation analysis was applied to examine the relationships among the PFCs.

\section{Results}

\subsection{Concentrations of PFCs in raw animal tissue sam- ples}

The levels of PFCs detected in raw animal tissue samples from Beijing are summarized in Tables 1 and 2. The mean levels of total PFCs were in the order pig liver $\left(3.438 \mathrm{ng} \mathrm{g}^{-1}\right.$ wet weight (ww))>pig kidney $\left(0.508 \mathrm{ng} \mathrm{g}^{-1}\right.$ (ww) $)>$ pig heart $\left(0.167 \mathrm{ng} \mathrm{g}^{-1}\right.$ (ww))>chicken liver $\left(0.098 \mathrm{ng} \mathrm{g}^{-1}(\mathrm{ww})\right)>$ chicken heart $\left(0.050 \mathrm{ng} \mathrm{g}^{-1}(\mathrm{ww})\right)>$ pork loin $\left(0.018 \mathrm{ng} \mathrm{g}^{-1}\right.$ $(w w)) \approx$ chicken breast $\left(0.012 \mathrm{ng} \mathrm{g}^{-1}\right.$ (ww)). The total PFCs level in pig liver was over 30-fold higher than that in chicken liver. However, the level in pig heart was only 3 -fold higher than that in chicken heart. Among these tissues, significantly lower levels of PFCs were observed in chicken breast and pork loin than other tissues. The PFCs, and in particular PFOS (0.094-11.30 $\mathrm{ng} \mathrm{g}^{-1}$ ), levels in pig liver samples were notably higher than in other tissue samples. Both PFOA and PFOS could be quantified in all of the

Table 1 Concentrations (ng g ${ }^{-1}$, wet weight) of perfluorinated compounds (PFCs) detected in various pig tissues ${ }^{\text {a) }}$

\begin{tabular}{|c|c|c|c|c|c|c|c|c|c|c|}
\hline & PFHxS & PFHpA & PFOA & PFOS & PFNA & PFDA & PFUnDA & PFDoDA & PFTA & Total PFCs \\
\hline \multicolumn{11}{|l|}{ Pig heart, $n=17$} \\
\hline Range & $<\mathrm{LOD}$ & $<$ LOD-0.062 & $<$ LOD-0.642 & $<$ LOD-0.136 & $<$ LOD-0.096 & $<$ LOD-0.068 & $<$ LOD-0.098 & $<$ LOD & $<\mathrm{LOD}$ & \multirow{4}{*}{0.167} \\
\hline Mean & & 0.008 & 0.107 & 0.024 & 0.009 & 0.005 & 0.014 & & & \\
\hline Median & & & 0.030 & 0.010 & & & & & & \\
\hline Positive (\%) & & 18 & 65 & 65 & 18 & 12 & 24 & & & \\
\hline \multicolumn{11}{|l|}{ Pig liver, $n=20$} \\
\hline Range & $<$ LOD-0.020 & $<$ LOD-0.060 & $0.034-1.790$ & $0.094-11.30$ & $<$ LOD-0.528 & $<$ LOD-0.614 & $<$ LOD -2.560 & $<$ LOD-0.536 & $<$ LOD-0.154 & \multirow{4}{*}{3.438} \\
\hline Mean & 0.002 & 0.012 & 0.241 & 2.163 & 0.161 & 0.150 & 0.560 & 0.124 & 0.025 & \\
\hline Median & & & 0.130 & 0.481 & 0.083 & 0.065 & 0.097 & & & \\
\hline Positive (\%) & 10 & 35 & 100 & 100 & 80 & 80 & 90 & 45 & 25 & \\
\hline \multicolumn{11}{|l|}{ Pig kidney, $n=24$} \\
\hline Range & $<\mathrm{LOD}$ & $<$ LOD-0.042 & $<$ LOD-4.979 & $<$ LOD- 0.718 & $<$ LOD-0.216 & $<$ LOD-0.071 & $<$ LOD-0.162 & $<$ LOD-0.037 & $<\mathrm{LOD}$ & \multirow{3}{*}{0.508} \\
\hline Mean & & 0.012 & 0.326 & 0.102 & 0.045 & 0.009 & 0.014 & & & \\
\hline Median & & & 0.059 & 0.055 & 0.030 & & & & & \\
\hline \multicolumn{11}{|l|}{ Pork loin, $n=20$} \\
\hline Range & $<\mathrm{LOD}$ & $<\mathrm{LOD}$ & $<$ LOD-0.097 & $<$ LOD-0.081 & $<\mathrm{LOD}$ & $<\mathrm{LOD}$ & $<$ LOD-0.043 & $<\mathrm{LOD}$ & $<\mathrm{LOD}$ & \multirow{3}{*}{0.018} \\
\hline Mean & & & 0.007 & 0.007 & & & 0.004 & & & \\
\hline Median & & & & & & & & & & \\
\hline Positive (\%) & & & 10 & 25 & & & 10 & & & \\
\hline
\end{tabular}

a) Mean values were not calculated when $>90 \%$ of the samples had values below the LOD. Median values are given when $>50 \%$ of the samples had values above the LOD. FOEA and FOUEA were not detected in any of the samples above LOD. 
Table 2 Concentrations ( $\mathrm{ng} \mathrm{g}^{-1}$, wet weight) of perfluorinated compounds (PFCs) detected in various chicken tissues ${ }^{\mathrm{a})}$

\begin{tabular}{|c|c|c|c|c|c|c|c|c|}
\hline & PFHpA & PFOA & PFOS & PFNA & PFDA & PFUnDA & PFDoDA & Total PFCs \\
\hline \multicolumn{9}{|c|}{ Chicken heart, $n=19$} \\
\hline Range & $<$ LOD-0.060 & $<$ LOD-0.031 & $<$ LOD- 0.273 & $<$ LOD-0.041 & $<$ LOD-0.043 & $<$ LOD-0.177 & $<$ LOD-0.034 & \multirow{4}{*}{0.050} \\
\hline Mean & 0.008 & 0.007 & 0.026 & 0.004 & 0.005 & & & \\
\hline Median & & & & & & & & \\
\hline Positive (\%) & 16 & 32 & 47 & 16 & 11 & 5 & 5 & \\
\hline \multicolumn{9}{|c|}{ Chicken liver, $n=19$} \\
\hline Range & $<$ LOD-0.034 & $<$ LOD-0.061 & $<$ LOD-0.288 & $<$ LOD-0.038 & $<$ LOD-0.042 & $<$ LOD-0.166 & $<$ LOD-0.039 & \multirow{4}{*}{0.098} \\
\hline Mean & 0.003 & 0.009 & 0.063 & 0.009 & 0.003 & 0.011 & & \\
\hline Median & & & 0.043 & & & & & \\
\hline Positive (\%) & 11 & 21 & 68 & 32 & 11 & 16 & 5 & \\
\hline \multicolumn{9}{|c|}{ Chicken breast, $n=24$} \\
\hline Range & $<$ LOD-0.017 & $<$ LOD-0.035 & $<$ LOD-0.137 & $<\mathrm{LOD}$ & $<\mathrm{LOD}$ & $<\mathrm{LOD}$ & $<\mathrm{LOD}$ & \multirow{4}{*}{0.012} \\
\hline Mean & & & 0.012 & & & & & \\
\hline Median & & & & & & & & \\
\hline Positive (\%) & 8 & 4 & 21 & & & & & \\
\hline
\end{tabular}

pig liver samples. Our data suggest that pigs may accumulate PFCs mainly in the liver. Additionally, PFHxS was only found in two of the twenty pig liver samples. In chicken liver, PFOS was the predominant compound, and was detected in $68 \%$ of the samples. In the other two chicken tissues sampled, PFOS was also the most frequently detected compound. The two fluorotelomer acids were not detected in any of the samples. In general, the levels of PFCs detected in chicken tissues were lower than those in pig tissues.

\subsection{Composition profile of PFCs}

The composition profiles of mean concentrations of the seven main PFCs in the tissues are shown in Figure 1. In pig liver, PFOS contributed up to $63 \%$ of the total composition of PFCs. Aside from PFOS, PFUnDA was the most prevalent PFC, contributing $16 \%$ of the total. The PFC-profile of pig kidney was very similar to that of pig heart, and PFOA was dominant, accounting for about two thirds of the total PFCs in the 2 tissues. In comparison, fewer PFCs were found in pork loin than pig viscera, with only 3 PFCs detected. In chicken liver and heart, PFOS contributed over half of the total PFCs. PFUnDA was observed in chicken liver, but not in chicken heart. In chicken breast samples, PFOS was the only compound detected.

\subsection{Correlations between PFCs in pig liver}

The levels of PFCs in pig liver were significantly higher than those in the other tissues and 5 PFCs (PFOA, PFOS, PFNA, PFDA and PFUnDA) were detected in over $80 \%$ of the samples. We found these five PFCs were significantly $(P<0.001-0.04)$ correlated with each other, except for PFOA/PFDA. The strongest linear correlation $(r=0.932)$ was observed between the concentrations of PFOS and

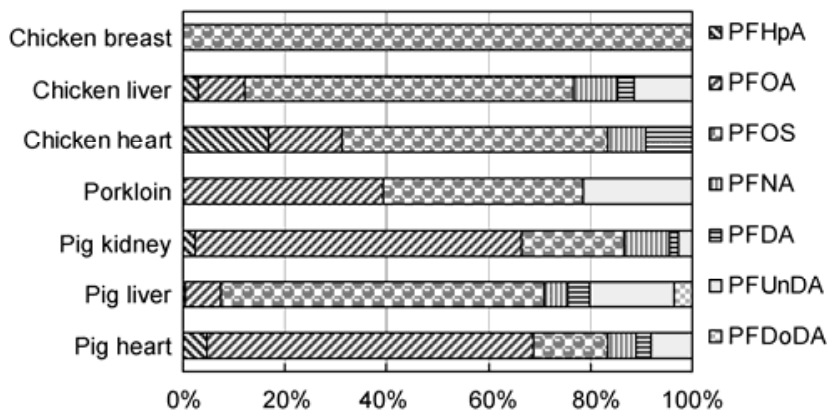

Figure 1 Composition profiles of the seven main perfluorinated compounds detected in pig and chicken tissue samples: perfluoroheptanoic acid (PFHpA), perfluorooctanoic acid (PFOA), perfluorooctane sulfonate (PFOS), perfluorononanoic acid (PFNA), perfluorodecanoic acid (PFDA), perfluoroundecanoic acid (PFUnDA), and perfluorododecanoic acid (PFDoDA).

PFUnDA (Figure 2), which indicated that exposure to these two PFCs might be from the same source. In pig kidney, PFOA and PFOS were detected in over $80 \%$ of the samples, but no statistically significant correlation $(P>0.05)$ was observed between them.

\section{Discussion}

\subsection{Comparison with other studies}

In general, the levels of PFCs observed in Beijing meat products were similar to or lower than those observed in other studies. In Japan, liver samples from farm animals (cattle, pigs, and chickens) were analyzed for 10 PFCs [23]. Among the analyzed PFCs, PFOS was dominant with mean liver concentrations of $67 \mathrm{ng} \mathrm{g}^{-1}$ in chickens, $54 \mathrm{ng} \mathrm{g}^{-1}$ in pigs, and $34 \mathrm{ng} \mathrm{g}^{-1}$ in cattle. The PFOS levels in pig and chicken livers in the Japanese study were much higher than in the corresponding samples in our study. In contrast, the levels of other PFCs were not significant and were compa- 


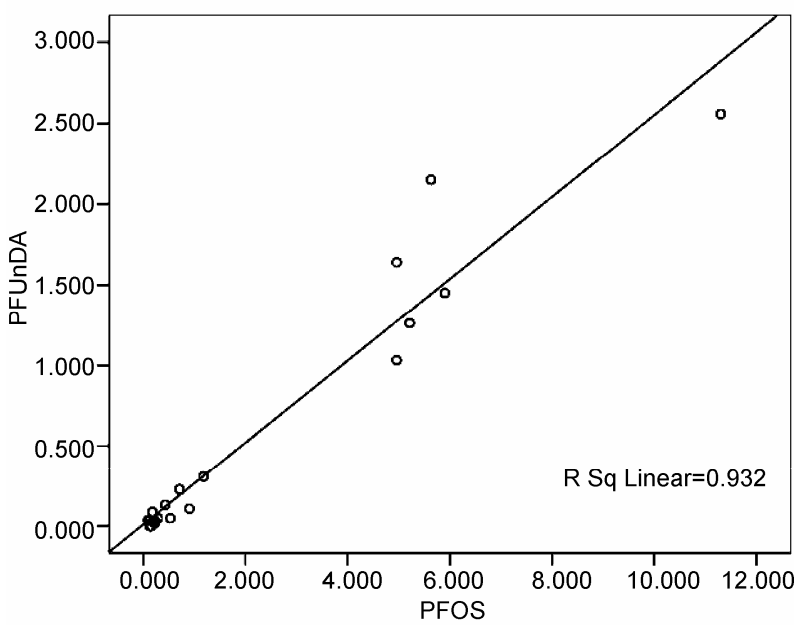

Figure 2 Correlation between perfluorooctane sulfonate (PFOS) and perfluoroundecanoic acid (PFUnDA) concentrations $\left(\mathrm{ng} \mathrm{g}^{-1}\right)$ in pig livers.

rable with those observed in our survey. It should be noted that the animals in the Japanese study had longer life spans (chickens 2.5-7 months, pigs 7 months) than in our study (chickens $40 \mathrm{~d}$, pigs 5 months) before slaughter.

In samples from Catalonia (Spain), levels of PFCs were determined in 36 composite samples of randomly purchased foodstuffs [20]. In pork and chicken samples, PFOS was the only compound detected. The mean concentration in pork $\left(0.045 \mathrm{ng} \mathrm{g}^{-1}\right)$ was somewhat higher than in our study, while the mean concentration in chicken $\left(0.02 \mathrm{ng} \mathrm{g}^{-1}\right)$ was consistent with our results. In another study from Catalonia, Jogsten et al. [27] determined 11 PFCs in composite samples of meat products including pork loin, chicken breast, pig liver and lamb liver. The results indicated that although PFOS was the most frequently detected compound (8/20 food items), the highest PFOS level was only $0.33 \mathrm{ng} \mathrm{g}^{-1}$ (fresh weight) in lamb liver. In comparison with our results, none of PFCs were above the LOD in pork loin, chicken breast or pig liver.

In the United Kingdom, 20 composite food groups from the 2004 Total Diet Study (TDS) were analyzed for 15 PFCs, including PFOS [28]. PFOS was found in only 4 food groups, and the remaining PFCs (0.5-20 $\mathrm{ng} \mathrm{g}^{-1}$ fresh weight) were detected in very few samples above the LOD. However, in the TDS, the LOD were notably higher than in our study. In Canada, Tittlemier et al. [18] analyzed 54 solid food composite samples of meat or other animal-derived food items for PFOS and perfluorocarboxylates. PFOS was predominant with a concentration of $2.7 \mathrm{ng} \mathrm{g}^{-1}$ (ww). In a recent study, the levels of nine PFCs were determined in the livers of 60 skipjack tuna (Katsuwonus pelamis) collected from offshore waters and the open ocean along the Pacific Rim [29]. Both PFOS and PFUnDA were predominant compounds at concentrations of $<1-58.9$ and $<1-31.6 \mathrm{ng} \mathrm{g}^{-1}$ (ww), respectively. The pig liver in our survey had similar composition profiles for these two PFCs. However, PFOA was rarely detected in the tuna livers, unlike the liver samples in our study.

\subsection{Dietary intake of PFCs through meat products}

In a recently published survey, the relative contributions of major food categories to food composition in Beijing were described [30]. Meat consumption for body weight (bw) was $1.6 \pm 1.3 \mathrm{~g} \mathrm{~kg}^{-1} \mathrm{~d}^{-1}$. On the basis of this value, we preliminarily estimated the dietary intake (DI) of PFCs through the consumption of meat products. Assuming pork is the only consumed meat product (not detected $=0$ ), the mean DI of PFOS and total PFCs are 0.011 and $0.029 \mathrm{ng} \mathrm{kg}^{-1} \mathrm{~d}^{-1}$, respectively. In a previous study, seven types of seafood collected from fish markets in two coastal cities of China were analyzed for nine PFCs [31]. The DI of PFOS and PFOA through fish consumption were $1.7-2.8$ and $1.9 \mathrm{ng} \mathrm{kg}^{-1} \mathrm{~d}^{-1}$, respectively. In comparison, the DI of PFCs via pork consumption is much lower. However, the DI of PFCs will increase when the consumption of animal viscera are taken into account. Although Chinese people do not consume much pig or chicken viscera, the levels of PFCs in viscera are much higher than those in muscle. The UK Food Standards Agency (UK FSA) Committee on Toxicology has established provisional tolerable daily intake (TDI) levels of 300 and $3000 \mathrm{ng}$ $\mathrm{kg}^{-1} \mathrm{~d}^{-1}$ for PFOS and PFOA, respectively [32,33]. The European Food Safety Authority (EFSA) has derived a TDI of $150 \mathrm{ng} \mathrm{kg}^{-1} \mathrm{~d}^{-1}$ for PFOS and $1500 \mathrm{ng} \mathrm{kg}^{-1} \mathrm{~d}^{-1}$ for PFOA [34]. Thus, for the population of Beijing, the DI of PFCs via meat products is far below the recommended values.

Some countries have estimated the DI of PFOS. The UK FSA study showed that the estimated average adult dietary intakes from the whole diet in 2004 were $100 \mathrm{ng} \mathrm{kg}^{-1} \mathrm{~d}^{-1}$ for PFOS and $70 \mathrm{ng} \mathrm{kg}^{-1} \mathrm{~d}^{-1}$ for PFOA [28]. In Catalonia, the DI was estimated to be $62.5 \mathrm{ng} \mathrm{kg}^{-1} \mathrm{~d}^{-1}(\mathrm{ND}=0)$ [20]. In Canada, the average DI of total perfluorocarboxylates and PFOS for Canadians was estimated to be $250 \mathrm{ng} \mathrm{kg}^{-1} \mathrm{~d}^{-1}$, based on results from the 2004 TDS composites study [18]. Another study from Canada showed that DI was 210-610 $\mathrm{ng} \mathrm{kg}{ }^{-1} \mathrm{~d}^{-1}$ in Nunavut [24]. Moreover, caribou meat contributed $43 \%-75 \%$ of daily dietary exposure to PFCs.

In summary, the levels of PFCs in animal viscera appeared higher than those in muscles. The levels in pig tissues were higher than those in the corresponding chicken tissues. Our results indicate that there is little potential risk of exposure to PFCs for the Beijing population via consumption of these products. However, further studies are required to investigate the source of PFCs in farmed animals. Additionally, the contribution of dietary intake to the total intake of PFCs should be investigated.

This work was supported by the National High-Technology Research and Development Program of China (2007AA06Z405), the Major Research Program of Chinese Academy of Sciences (KZCX2-YW-420-1), the National Natural Science Foundation of China (20907063, 20837003, 20890111) and the state key Basic Research Program of China (2009CB421605).

1 Prevedouros K, Cousins I T, Buck R C, et al. Sources, fate and transport of perfluorocarboxylates. Environ Sci Technol, 2006, 40: $32-44$ 
2 Wang T, Wang Y W, Liao C Y, et al. Perspectives on the inclusion of perfluorooctane sulfonate into the Stockholm Convention on Persistent Organic Pollutants. Environ Sci Technol, 2009, 43: 5171-5175

3 Houde M, Martin J W, Letcher R J, et al. Biological monitoring of polyfluoroalkyl substances: A review. Environ Sci Technol, 2006, 40: 3463-3473

4 Senthilkumar K, Ohi E, Sajwan K, et al. Perfluorinated compounds in river water, river sediment, market fish, and wildlife samples from Japan. Bull Environ Contam Toxicol, 2007, 79: 427-431

5 Suja F, Pramanik B K, Zain S M. Contamination, bioaccumulation and toxic effects of perfluorinated chemicals (PFCs) in the water environment: a review paper. Water Sci Technol, 2009, 60: 1533-1544

6 Vestergren R, Cousins I T. Tracking the pathways of human exposure to perfluorocarboxylates. Environ Sci Technol, 2009, 43: 5565-5575

7 Liu W, Dong G H, Jin Y H, et al. Occurrence of perfluoroalkyl acids in precipitation from Shenyang, China. Chinese Sci Bull, 2009, 54: 2440-2445

8 Ropers M H, Durand S, Veyrand B, et al. Contamination of food by fluorinated surfactants-Distribution in emulsions and impact on the interfacial protein behaviour. Food Hydrocolloid, 2009, 23: 1149-1155

9 Ahrens L, Siebert U, Ebinghaus R. Total body burden and tissue distribution of polyfluorinated compounds in harbor seals (Phoca vitulina) from the German Bight. Mar Pollut Bull, 2009, 58: 520-525

10 Kannan K, Koistinen J, Beckmen K, et al. Accumulation of perfluorooctane sulfonate in marine mammals. Environ Sci Technol, 2001, 35: 1593-1598

11 Houde M, Bujas T A D, Small J, et al. Biomagnification of perfluoroalkyl compounds in the bottlenose dolphin (Tursiops truncatus) food web. Environ Sci Technol, 2006, 40: 4138-4144

12 Conder J M, Hoke R A, De Wolf W, et al. Are PFCAs bioaccumulative? A critical review and comparison with regulatory lipophilic compounds. Environ Sci Technol, 2008, 42: 995-1003

13 De Vos M G, Huijbregts M A J, Van Den Heuvel-Greve M J, et al. Accumulation of perfluorooctane sulfonate (PFOS) in the food chain of the Western Scheldt estuary: Comparing field measurements with kinetic modeling. Chemosphere, 2008, 70: 1766-1773

14 Yeung L W Y, So M K, Jiang G B, et al. Perfluorooctanesulfonate and related fluorochemicals in human blood samples from China. Environ Sci Technol, 2006, 40: 715-720

15 Longnecker M P, Smith C S, Kissling G E, et al. An interlaboratory study of perfluorinated alkyl compound levels in human plasma. Environ Res, 2008, 107: 152-159

16 Kubwabo C, Stewart B, Zhu J P, et al. Occurrence of perfluorosulfonates and other perfluorochemicals in dust from selected homes in the city of Ottawa, Canada. J Environ Monit, 2005, 7: 1074-1078

17 Tittlemier S A, Pepper K, Edwards L, et al. Concentrations of perfluorooctanesulfonamides in Canadian total diet study composite food samples collected between 1992 and 2004. J Agric Food Chem, 2006, 54: 8385-8389

18 Tittlemier S A, Pepper K, Seymour C, et al. Dietary exposure of Canadians to perfluorinated carboxylates and perfluorooctane sulfonate via consumption of meat, fish, fast foods, and food items prepared in their packaging. J Agric Food Chem, 2007, 55: 3203-3210
19 Fromme H, Schlummer M, Moller A, et al. Exposure of an adult population to perfluorinated substances using duplicate diet portions and biomonitoring data. Environ Sci Technol, 2007, 41: 7928-7933

20 Ericson I, Mart-Cid R, Nadal M, et al. Human exposure to perfluorinated chemicals through the diet: Intake of perfluorinated compounds in foods from the Catalan (Spain) market. J Agric Food Chem, 2008, 56: 1787-1794

21 Halldorsson T I, Fei C, Olsen J, et al. Dietary predictors of perfluorinated chemicals: A study from the Danish national birth cohort. Environ Sci Technol, 2008, 42: 8971-8977

22 Mak Y L, Taniyasu S, Yeung L W Y, et al. Perfluorinated compounds in tap water from China and several other countries. Environ Sci Technol, 2009, 43: 4824-4829

23 Guruge K S, Manage P M, Yamanaka N, et al. Species-specific concentrations of perfluoroalkyl contaminants in farm and pet animals in Japan. Chemosphere, 2008, 73: S210-S215

24 Ostertag S K, Tague B A, Humphries M M, et al. Estimated dietary exposure to fluorinated compounds from traditional foods among Inuit in Nunavut, Canada. Chemosphere, 2009, 75: 1165-1172

25 Zhai F Y, Wang H J, Du S F, et al. A prospective study on nutrition transition in China (in Chinese). J Med Res, 2006, 35: 3-7

26 Taniyasu S, Kannan K, So M K, et al. Analysis of fluorotelomer alcohols, fluorotelorner acids, and short- and long-chain perfluorinated acids in water and biota. J Chromatogr A, 2005, 1093: 89-97

27 Jogsten I E, Perello G, Llebaria X, et al. Exposure to perfluorinated compounds in Catalonia, Spain, through consumption of various raw and cooked foodstuffs, including packaged food. Food Chem Toxicol, 2009, 47: 1577-1583

28 UK FSA (U.K. Food Standards Agency). Fluorinated Chemicals: U.K. Dietary Intakes. Food Survey Information Sheet 11/06, 2006, available at http://www.food.gov.uk/science/surveillance/fsisbranch2006/ fsis 1106

29 Hart K, Kannan K, Tao L, et al. Skipjack tuna as a bioindicator of contamination by perfluorinated compounds in the oceans. Sci Total Environ, 2008, 403: 215-221

30 Yu Y, Tao S, Liu W, et al. Dietary intake and human milk residues of hexachlorocyclohexane isomers in two Chinese cities. Environ Sci Technol, 2009, 43: 4830-4835

31 Gulkowska A, Jiang Q T, So M K, et al. Persistent perfluorinated acids in seafood collected from two cities of China. Environ Sci Technol, 2006, 40: 3736-3741

32 COT (Committee on Toxicity of Chemicals in Food, Consumer Products and the Environment), 2006a. COT statement on the tolerable daily intake for perfluorooctane sulfonate. COT statement 2006/09, 2006, available at http://cot.food.gov.uk/pdfs/cotstatementpfos200609.pdf

33 COT (Committee on Toxicity of Chemicals in Food, Consumer Products and the Environment), 2006b. COT statement on the tolerable daily intake for perfluorooctanoic acid. COT statement 2006/10, 2006, available at http://cot.food.gov.uk/pdfs/cotstatementpfoa200610.pdf

34 EFSA. Perfluorooctane sulfonate (PFOS), perfluorooctanoic acid (PFOA) and their salts. Scientific opinion of the panel on contaminants in the food chain, 2008, available at http://www.efsa.europa.eu/EFSA/ efsa_locale-1178620753812_1211902012410.htm

\section{Supporting Information}

Figure S1 Fourteen sampling sites (red pentagrams) in the downtown of Beijing

Table S1 Experimental conditions of electrospray tandem mass spectrometry

Table S2 Recovery results $(n=3)$ for the various matrices

The supporting information is available online at csb.scichina.com and www.springerlink.com. The supporting materials are published as submitted, without typesetting or editing. The responsibility for scientific accuracy and content remains entirely with the authors. 